\title{
Lipase catalysed resolution and microbial reduction for obtaining enantiopure 1-(2-thienyl)alkanols
}

\author{
Eirik Sundby1, Morten Myhre Andersen1, Bård Helge Hoff 2 and \\ Thorleif Anthonsen1* \\ ${ }^{1}$ Department of Chemistry, Norwegian University of Science and Technology, N-7491 \\ Trondheim, Norway \\ ${ }^{2}$ Borregaard Synthesis, P.O. Box 100, N-1701 Sarpsborg \\ E-mail: Thorleif.Anthonsen@chembio.ntnu.no
}

\section{Dedicated to Professor Kjell Undheim on the Occasion of his $70^{\text {th }}$ birthday (received 12 Jul 01; accepted 24 Mar 02; published on the web 01 Apr 02)}

\begin{abstract}
Efficient methods have been developed for resolving 1-(2-thienyl)alkanols with lipase B from Candida antarctica as catalyst. Fermenting cells and cell-free reduction systems of Geotrichum candidum (IFO 4597) have also been tested in asymmetric reductions of 1-(2-thienyl)alkan-1ones.
\end{abstract}

Keywords : Lipase, resolution, 1-(2-thienyl)alkanols, microbial reduction

\section{Introduction}

Kinetic resolution is an efficient method for providing both enantiomers of chiral compounds. The success of a process depends profoundly on the ability of the enzyme to select between the two enantiomers as expressed by the enantiomeric ratio $\mathrm{E}$, which is the relative rate of reaction with the two enantiomers. ${ }^{1}$ Compared to asymmetric synthesis, kinetic resolution has the benefit that a high enantiomeric excess can be obtained even with a moderate E-value. One of the short comings of lipase catalysed resolutions is that the maximum yield is limited to $50 \%$. This can be overcome for instance by performing a dynamic kinetic resolution in which the unreacted enantiomer is continuously racemised.

Various micro-organisms have been used for oxidative kinetic resolution of 1-(2thienyl)ethanol. ${ }^{2-4}$ 1-(2-Thienyl)propanol has previously been resolved with Pseudomonas cepacia as catalyst with moderate E-values (15-30) depending on temperature. ${ }^{5}(R)-1-(2-$ Thienyl)ethanol and (R)-1-(2-thienyl)propanol have been prepared with ee of $91 \%$ and $88 \%$ respectively using an oxazaborolidine catalyst. ${ }^{6}(R)-1-(2-$ Thienyl)ethanol has also been reported 
via a Rhodium-catalysed asymmetric hydrosilylation in good yields and $76 \%$ ee. $^{7}$

\section{Results and Discussion}

\section{Synthesis of starting compounds}

The ketones 2a-4a were synthesised by a modified Friedel-Crafts acylation of thiophene ${ }^{8}$, using $\mathrm{SnCl}_{4}$ as the Lewis acid as shown in Scheme 1 (1a was purchased). Thiophene was reacted with the appropriate acid chloride and the yield was 80-90\%. The corresponding ketones were reduced with sodium borohydride to give the racemic alcohols in good yields.

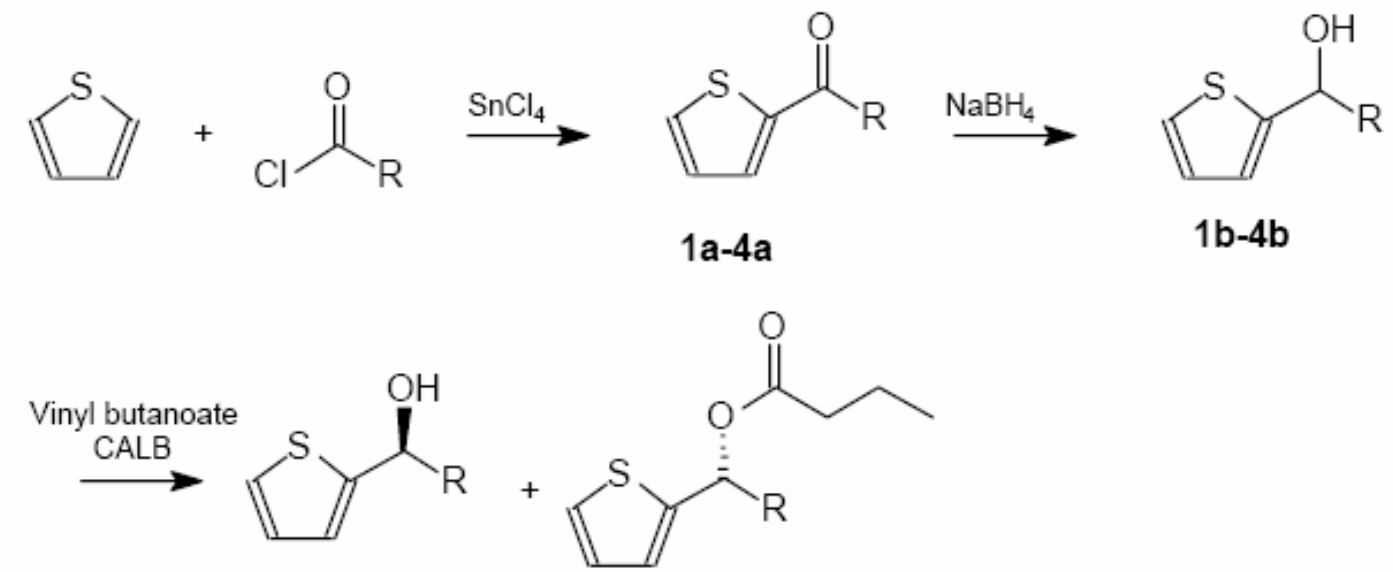

(S)-1b-4b

(R)-1c-4c
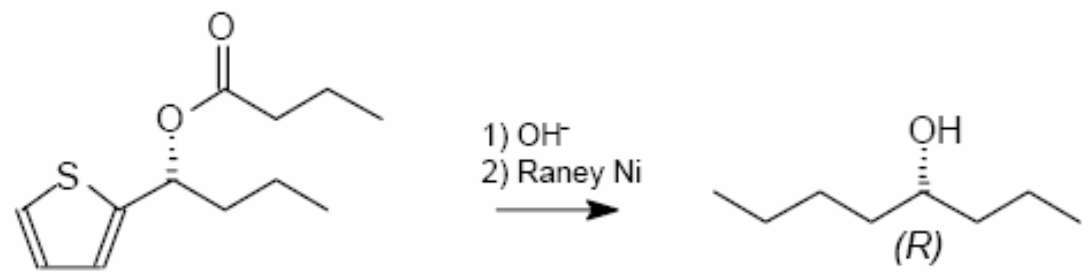

(R)

(R)-3c

Scheme 1. Synthesis of 2-acyl thiophenes, reduction to 1-(2-thienyl)-1-alkanols and lipase catalysed resolutions, $\mathrm{R}=\mathrm{Me}(\mathbf{1}), \mathrm{Et}(2), \mathrm{Pr}(3), \mathrm{Bu}$ (4). In order to prove the absolute configuration of $3 \mathrm{c}$, it was converted to $(R)-4$-octanol.

\section{Lipase catalysis}

The four secondary alcohols were resolved in a series of solvents with lipase B from Candida antarctica (CALB) as catalyst. Vinyl butanoate was used as acyl donor.

CALB displayed high E-values for all secondary alcohols. We have previously explored the substrate requirements of this enzyme and found that in organic solvents an ethyl group is the largest of the "small" substituent (in the present substrates the "large" substituent is the 
thiophene moiety) of a secondary alcohol/ester in order to obtain a high E-value ${ }^{9}$. However, for the present thiophenes, also $\mathrm{R}=$ propyl or butyl also gave very high E-values (Table 1).

Table 1. E-values of CALB catalysed kinetic resolution of 1-(2-thienyl)alkanols

$$
\mathrm{R} \text { Hexane Benzene } \mathrm{Et}_{2} \mathrm{O} \text { Dioxane Toluene t.-BuOMe } \mathrm{CCl}_{4}
$$

\begin{tabular}{ccccccccc}
\hline $\mathbf{1 b}$ & $\mathrm{Me}$ & 204 & 211 & 221 & 167 & 236 & 217 & 146 \\
$\mathbf{2 b}$ & $\mathrm{Et}$ & $>500$ & $>500$ & $>500$ & 382 & $>500$ & $>500$ & $>500$ \\
$\mathbf{3 b}$ & $\mathrm{Pr}$ & 148 & $>500$ & 216 & 176 & 218 & 369 & 349 \\
$\mathbf{4 b}$ & $\mathrm{Bu}$ & 196 & 150 & 152 & 73 & 237 & 90 & 66 \\
\hline
\end{tabular}

\section{Stereo preference of resolutions}

For both 1-(2-thienyl)ethanol (1b) and 1-(2-thienyl)propanol (2b), the (R)-enantiomer was the faster reacting in the transesterifications, shown by comparing the optical rotation of the remaining alcohol with previously reported values. ${ }^{2-5}$ Since no optical rotation has been reported for enantiopure 1-(2-thienyl)butanol (3b) or the corresponding butanoate, the ester was hydrolysed and treated with Raney-Ni to give (R)-4-octanol10 with $[\alpha]_{D}^{20}=+0.25^{\circ}$ This shows that the $(R)$-butanoate also was formed in this case. This way of proving the configuration is not applicable for 1-(2-thienyl)pentanol (4b) since the formed secondary alcohol is achiral. However, based on knowledge of the stereo preference of CALB and and the elution order of enantiomeric alcohols and esters (Table 2), it is reasonable to assume that the $(R)$-ester and the $(S)$-alcohol are the products also in the resolution of $\mathbf{4} \mathbf{b}^{11}$.

In order to further prove the stereopreference in the transesterifications, we subjected the remaining ester $[(R)-1 c]$ to enzyme catalysed methanolysis. The produced alcohol gave $[\alpha]_{20} \mathrm{D}_{=}$ $+21.66^{\circ}$, almost exactly opposite the reported values for the (S)-alcohol.

\section{Microbial asymmetric reduction}

The ketones 1a-4a were subject either to fermenting cells or a cell free acetone powder12 of Geotrichum candidum (IFO 4597). Only 1a was reduced to give (S)-1b. This is in agreement with earlier reported substrate requirements for the oxido-reductases of Geotrichum candidum.12 Fermenting cells gave an ee of $74 \%$, but high ee's (>99\%) were obtained using acetone powder in buffer. However, the yields were not optimal, being $54 \%$ in MES buffer and $78 \%$ in phosphate buffer after $24 \mathrm{~h}$. We tried to improve this by performing the reduction in organic solvents using $G$. candidum cells immobilized on Celite in hexane. Unfortunately this failed to give any measurable reduced product for any of the substrates 1a-4a. A different system consisting of fermenting cells of G. candidum, immobilised on the water-absorbing polymer LP100, also failed to give reductions for the substrates 2a-4a. However, 1a was reduced in $7 \%$ yield after 4 days. This was considered too low to be of practical importance and further attempts were not carried out. 


\section{Experimental Section}

General Procedures. Immobilized CALB (Novozyme 435, Novozymes ) had an activity of $7000 \mathrm{PLU} / \mathrm{g}$, and a water content of $1-2 \% \mathrm{w} / \mathrm{w}$. Solvents were dried over molecular sieves. The cultivation of Geotrichum candidum (IFO 4597) and preparations of its acetone-powder (AGP) are described elsewhere. ${ }^{12}$ Column chromatography was performed using silica gel 60 from Fluka. Enzymatic reactions were performed in a shaker incubator (New Brunswick, Edison, NJ, USA). 1-(2-Thienyl)ethan-1-one (1a) was purchased from Fluka and the water absorbing polymer LP-100 via Sigma, Norway.

\section{Analyses}

Optical rotations were determined using an Optical Activity Ltd. AA-10 automatic polarimeter, concentrations are given in $\mathrm{g} / 100 \mathrm{~mL}$. NMR spectra were recorded in $\mathrm{CDCl}_{3}$ solutions, using Bruker DPX 300 and 400 instruments, operating at 300 and $400 \mathrm{MHz}$ for ${ }^{1} \mathrm{H}$ and 75 and 100 $\mathrm{MHz}$ for ${ }^{13} \mathrm{C}$, respectively. Chemical shifts are in ppm relative to TMS and coupling constants in Hz. Enantiomeric ratios, E were calculated on the basis of measurement of enantiomeric excess of both substrate (ees) and product (eep) at several degrees of conversion, using the computer program E \& K calculator version 2.03 ${ }^{13}$. Mass spectra were recorded on a Fison 8065/Fison TRIO 1000 GC-MS (EI, 70eV) system with a quadrupole mass filter, equipped with a DB-1701 column from J\&W Scientific ( $25 \mathrm{~m}, 0.25 \mathrm{~mm}$ ID, $0.25 \mu \mathrm{m}$ film thickness). Helium was used as carrier gas. Chiral analyses were performed using a Varian 3400 or 3800 gas chromatograph from Varian Instrument Group, Walnut Creek, California, USA. The columns were either Chiraldex G-TA, $10 \mathrm{~m}, 0.25 \mathrm{~mm}$ ID, $0.25 \mu \mathrm{m}$ film thickness from Astec, Whippany, N.J., USA or CP-Chirasil-Dex CB, $25 \mathrm{~m}, 0.25 \mathrm{~mm}$ ID, $0.25 \mu \mathrm{m}$ film thickness from Chrompack, Chrompack Norge A.S., Sandvika, Norway. The gas chromatographs were pressure regulated, carrier gas was hydrogen (Hydrogen 5.0, purity: 99.999\%) with an outlet pressure of 3 bar (7 psi). Injection was performed in split mode at $200^{\circ} \mathrm{C}$, split ratios 60 . Detection was done using FID detectors at $200^{\circ} \mathrm{C}$ with air $(300 \mathrm{~mL} / \mathrm{min})$ and hydrogen $(30 \mathrm{~mL} / \mathrm{min})$ as flame gases. Integration was performed with Varian Star 4.0/4.5. The chromatographic details are given in Table 2. 
Table 2. Columns, temperature programs and chromatographic properties of alcohols $\mathbf{1} \mathbf{b}-\mathbf{4 b}$ and butanoates 1c-4c

\begin{tabular}{cccccc}
\hline & Column & $\begin{array}{c}\text { Temp. prog. } \\
\text { Temp. increase } 15^{\circ} \mathrm{C} / \mathrm{min}\end{array}$ & $\mathrm{t}_{\mathrm{S}}$ & $\mathrm{t}_{\mathrm{R}}$ & $\mathrm{R}_{\mathrm{S}}$ \\
\hline 1b & Chirasil-dex & $110{ }^{\circ} \mathrm{C}(11 \mathrm{~min}), 130{ }^{\circ} \mathrm{C}$ & 11.84 & 12.34 & 2.55 \\
1c & Chirasil-dex & $110^{\circ} \mathrm{C}(10 \mathrm{~min}), 180{ }^{\circ} \mathrm{C}$ & 14.91 & 14.68 & 2.16 \\
2b & Chiraldex GT-A & $90^{\circ} \mathrm{C}(10 \mathrm{~min}), 150{ }^{\circ} \mathrm{C}$ & 8.60 & 9.22 & 3.10 \\
2c & Chiraldex GT-A & $130{ }^{\circ} \mathrm{C}(10 \mathrm{~min}), 180{ }^{\circ} \mathrm{C} \#$ & 12.42 & 12.62 & 1.34 \\
3b & Chirasil-dex & $130{ }^{\circ} \mathrm{C}(10 \mathrm{~min}), 180{ }^{\circ} \mathrm{C}$ & 9.39 & 9.73 & 1.90 \\
3c & Chiraldex GT-A & $90^{\circ} \mathrm{C}(10 \mathrm{~min}), 150{ }^{\circ} \mathrm{C}$ & 7.64 & 8.04 & 1.86 \\
4b & Chiraldex GT-A & $80^{\circ} \mathrm{C}(20 \mathrm{~min}), 150{ }^{\circ} \mathrm{C} \#$ & 22.12 & 23.34 & 1.35 \\
4c & Chiraldex GT-A & $85^{\circ} \mathrm{C}(30 \mathrm{~min}), 150{ }^{\circ} \mathrm{C} \#$ & 32.54 & 34.02 & 1.23 \\
\hline
\end{tabular}

${ }^{*}$ Much effort was put into getting baseline separation. This was not achived in these cases.

\section{Immobilisation and enzymatic reduction in hexane AGP-celite}

Celite was prepared washing with EtOH $(2 \mathrm{x})$ and then distilled water until the upper water was clear. AGP (100 mg) and NAD+ $(20 \mathrm{mg})$ were suspended in MES buffer (2- $(N-$ morpholino)ethanesulphonic acid-NaOH) $(0.1 \mathrm{M}, \mathrm{pH} 7.0,3.0 \mathrm{~mL})$ and left for hydration for 15 min, then mixed with the celite. The preparation was dried under vacuum overnight. Each reaction was performed in hexane $(4 \mathrm{~mL})$ by adding the immobilized APG $(0.5 \mathrm{~g})$, 2-propanol $(100 \mu \mathrm{L})$ and substrate $(20 \mathrm{mg})$.

\section{Fermenting cells-LP-100}

Geotrichum candidum was grown in $1 \mathrm{~L}$ standard medium and filtered after $36 \mathrm{~h}$ to give $25 \mathrm{~g}$ of wet cells. ${ }^{12}$ The cells were re-suspended in distilled water $(100 \mathrm{~mL})$ and the water-absorbing polymer (LP-100, $15 \mathrm{~g}$ ) was added. Each reaction was performed in hexane $(6 \mathrm{~mL})$ by adding the immobilised cells ( $4 \mathrm{~g}$ ) and substrate $(20 \mathrm{mg})$.

\section{Small scale transesterifications}

Substrate alcohol $\left(2.2 \times 10^{-4}\right.$ mole) was dissolved in solvent $(3 \mathrm{~mL})$, vinyl butanoate $(5$ equivalents) was added and the reaction was started by adding immobilised CALB (10 $\mathrm{mg}$ ) to the reaction mixture at $30^{\circ} \mathrm{C}$. Chiral GLC analysis gave the enantiomeric excess of substrate (ees) and product (eep) from which conversion, c, was calculated, c = ees / ( ees + eep). In control experiments without enzyme, no acylation was observed using vinyl butanoate as acyl donor.

\section{Enzymatic reduction in water (small scale)}

The ketones 1a-4a $(0.013$ mmole $), \mathrm{NAD}+(7 \mu \mathrm{mole})$ and cyclopentanol or 2-propanol $(100 \mu \mathrm{L})$ were added to a suspension of AGP $(20 \mathrm{mg})$ in MES buffer, $(0.1 \mathrm{M}, \mathrm{pH} 7.0,3 \mathrm{~mL})$ or phosphate buffer $(0.1 \mathrm{M}, \mathrm{pH} 7.0,3 \mathrm{~mL})$. The mixture was shaken at $180 \mathrm{rpm}$ at $30{ }^{\circ} \mathrm{C}$. Small aliquots were withdrawn and extracted with $\mathrm{Et}_{2} \mathrm{O}$ for GLC analyses. When GLC showed maximum yield, the 
biomass was filtered off and filtrate saturated with $\mathrm{NaCl}$, before extracting with $\mathrm{Et}_{2} \mathrm{O}$. The combined extracts were dried with $\mathrm{MgSO}_{4}$ and evaporated. In control experiments without cofactor, no reduction was observed.

\section{Reduction by fermenting cells}

Geotrichum candidum was grown for 24 hours in $250 \mathrm{~mL}$ standard medium as described elswehere.12 The subtrates (100 mg) was added to $1 \mathrm{~mL}$ of ethanol, added to the cultures and incubation continued using the same conditions. Progress of the reactions was measured by TLC. Workup and analysis was equal to the reduction by AGP in water.

1-(2-Thienyl)ethan-1-one (1a). 1-(2-Thienyl)ethan-1-one (1a) was obtained from Fluka. ${ }^{1} \mathrm{H}$ NMR $\delta 2.55\left(3 \mathrm{H}, \mathrm{s}, \mathrm{COCH}_{3}\right), 7.11\left(1 \mathrm{H}, \mathrm{dd},{ }^{3} \mathrm{~J}=3.85\right.$ and $4.82 \mathrm{~Hz}$., thienyl H4), $7.62\left(\mathrm{~d}, 1 \mathrm{H},{ }^{3} \mathrm{~J}=\right.$ $3.85 \mathrm{~Hz}$ thienyl $\mathrm{H} 3)$ and $7.70\left(\mathrm{~d}, 1 \mathrm{H},{ }^{3} \mathrm{~J}=4.82 \mathrm{~Hz}\right.$, thienyl $\left.\mathrm{H} 5\right)$ ppm. ${ }^{13} \mathrm{C}$ NMR $\delta 26.7128 .5$ 132.5133 .8144 .5 and 190.7 ppm. MS: $126\left(\mathrm{M}^{\circ+}\right), 111(100 \%), 83,69,57,45,43$.

1-(2-Thienyl)propan-1-one (2a). Dry thiophene (9.3 mL, 0.118 mole), propanoic chloride (10.3 $\mathrm{mL}, 0.118$ mole) and dry benzene $(121.8 \mathrm{~mL})$ was mixed and cooled to $0{ }^{\circ} \mathrm{C}$. $\mathrm{SnCl}_{4}(13.96 \mathrm{~mL}$, 0.118 mole) was added dropwise over a $2 \mathrm{~h}$ period. The cooling was then removed and the stirring continued for another hour. To the reaction mixture was then added $\mathrm{HCl}$ (conc, $6 \mathrm{~mL}$ ) and ice water $(55 \mathrm{~mL})$. The organic layer was separated, the water phase extracted with $\mathrm{CH}_{2} \mathrm{Cl}_{2}$, the combined organic phase washed with water, dried with $\mathrm{MgSO}_{4}$ and evaporated. The product was purified by column chromatography (pentane : $\mathrm{Et}_{2} \mathrm{O}, 3: 1$ ) giving $2 \mathrm{a}$ in $91 \%$ yield. ${ }^{1} \mathrm{H}$ NMR $\delta 1.23\left(3 \mathrm{H}, \mathrm{t},{ }^{3} \mathrm{~J}=7.4 \mathrm{~Hz}, \mathrm{CH}_{2} \mathrm{CH}_{3}\right), 2.94\left(2 \mathrm{H}, \mathrm{q}, \mathrm{COCH}_{2}\right), 7.11\left(1 \mathrm{H}, \mathrm{dd},{ }^{3} \mathrm{~J}=3.85\right.$ and $5.00 \mathrm{~Hz}$, thienyl H4), $7.60\left(\mathrm{~d}, 1 \mathrm{H},{ }^{3} \mathrm{~J}=5.00 \mathrm{~Hz}\right.$, thienyl H5) and $7.69\left(\mathrm{~d}, 1 \mathrm{H},{ }^{3} \mathrm{~J}=3.85 \mathrm{~Hz}, \mathrm{H} 3\right)$ ppm. ${ }^{13} \mathrm{C}$ NMR $\delta 8.9,32.9,128.7,131.9,133.6,144.5$, and 194.2 ppm. MS: $140\left(\mathrm{M}^{\bullet+}\right), 111$ $(100 \%), 83,69,57,45$. TLC; $\mathrm{R}_{\mathrm{f}}=0.37$ (pentane : $\mathrm{Et}_{2} \mathrm{O}, 3: 1$ ).

1-(2-Thienyl)butan-1-one (3a). 1-(2-Thienyl)butan-1-one (3a) was synthesised by the same method as 2a. Yield: $84.7 \%$. ${ }^{1} \mathrm{H}$ NMR $\delta 1.00\left(3 \mathrm{H}, \mathrm{t},{ }^{3} \mathrm{~J}=7.4 \mathrm{~Hz}, \mathrm{CH}_{2} \mathrm{CH}_{3}\right), 1.79(2 \mathrm{H}, \mathrm{m}$, $\left.\mathrm{CH}_{2} \mathrm{CH}_{3}\right), 2.84\left(2 \mathrm{H}, \mathrm{t},{ }^{3} \mathrm{~J}=7.4 \mathrm{~Hz}, \mathrm{COCH}_{2}\right), 7.12\left(\mathrm{dd}, 1 \mathrm{H},{ }^{3} \mathrm{~J}=3.8\right.$ and $4.9 \mathrm{~Hz}$, thienyl H4), 7.61 $\left(\mathrm{d}, 1 \mathrm{H},{ }^{3} \mathrm{~J}=3.8\right.$, thienyl $\mathrm{H} 3$ ) and $7.71\left(\mathrm{~d}, 1 \mathrm{H}\right.$, and ${ }^{3} \mathrm{~J}=4.9 \mathrm{~Hz}$, thienyl H5) ppm. ${ }^{13} \mathrm{C}$ NMR: 13.8 , 18.2, 41.3, 128.3, 131.6, 133.3, 144.6 and 192.5 ppm. MS: $154\left(\mathrm{M}^{*+}\right), 126,111(100 \%), 83,57$, 45. $\mathrm{TLC} ; \mathrm{R}_{\mathrm{f}}=0.37$ (pentane : Et2O, $3: 1$ ).

1-(2-Thienyl)pentane-1-one (4a). 1-(2-Thienyl)pentane-1-one (4a) was synthesised by the same method as 2a. Yield: $72.4 \%$. ${ }^{1} \mathrm{H}$ NMR $\delta 0.94\left(3 \mathrm{H}, \mathrm{t},{ }^{3} \mathrm{~J}=7.4 \mathrm{~Hz}, \mathrm{CH}_{2} \mathrm{CH}_{3}\right), 1.40(2 \mathrm{H}, \mathrm{m}$, $\left.\mathrm{CH}_{2} \mathrm{CH}_{3}\right), 1.72\left(2 \mathrm{H}, \mathrm{m}, \mathrm{CH}_{2} \mathrm{CH}_{2} \mathrm{CH}_{3}\right) 2.88\left(2 \mathrm{H}, \mathrm{t},{ }^{3} \mathrm{~J}=7.3 \mathrm{~Hz}, \mathrm{COCH}_{2}\right), 7.10\left(1 \mathrm{H}, \mathrm{dd},{ }^{3} \mathrm{~J}=3.8\right.$ and $4.9 \mathrm{~Hz}$, thienyl $\mathrm{H} 4), 7.58\left(\mathrm{~d},{ }^{3} \mathrm{~J}=3.8 \mathrm{~Hz}, 1 \mathrm{H}\right.$, thienyl $\left.\mathrm{H} 5\right)$ and $7.69\left(\mathrm{~d},{ }^{3} \mathrm{~J}=3.8 \mathrm{~Hz}, 1 \mathrm{H}\right.$ thienyl H3) ppm. ${ }^{13} \mathrm{C}$ NMR $\delta 13.9,22.6,26.9,39.3,128.0,131.2,132.9144 .5$ and 193.5 ppm. MS: $168\left(\mathrm{M}^{\bullet+}\right), 139,126,111(100 \%), 83,57,41 . \mathrm{TLC} ; \mathrm{R}_{\mathrm{f}}=0.37$ (pentane : $\mathrm{Et}_{2} \mathrm{O}, 3: 1$ ).

The racemic secondary alcohols $\mathbf{1 b}-\mathbf{4 b}$ were synthesised from the ketones $\mathbf{1 a}-\mathbf{4 a}$ by $\mathrm{NaBH}_{4}$ reduction under standard conditions, quenching the reaction with $\mathrm{NH}_{4} \mathrm{Cl}^{14}$.

1-(2-Thienyl)ethanol (1b). Yield: $90.3 \% .{ }^{1} \mathrm{H}$ NMR $\delta$ (in accordance with previously reported spectra $\left.^{6}\right) 1.57\left(\mathrm{~d}, 3 \mathrm{H},{ }^{3} \mathrm{~J}=6.2 \mathrm{~Hz}, \mathrm{CHCH}_{3}\right), 5.11\left(\mathrm{q}, 1 \mathrm{H},{ }^{3} \mathrm{~J}=6.2 \mathrm{~Hz}, \mathrm{CHCH}_{3}\right), 6.93-7.0(\mathrm{~m}, 2 \mathrm{H}$, thienyl $\mathrm{H} 3$ and $\mathrm{H} 5)$ and 7.20-7.25 (m, 1H, thienyl H4) ppm. ${ }^{13} \mathrm{C}$ NMR $\delta 25.6,66.1,123.1,124.3$, 
126.6 and 150.0 ppm. MS: $128\left(\mathrm{M}^{\bullet+}\right), 113,110,95,85(100 \%), 66,58,45$. TLC; $\mathrm{R}_{\mathrm{f}}=0.19$ (EtOAc : hexane, $1: 5$ ).

1-(2-Thienyl)propanol (2b). Yield: $75.5 \%$. ${ }^{1} \mathrm{H}$ NMR $\delta$ (in accordance with previously reported spectra5) 0.94, (t, 3H, $\left.{ }^{3} \mathrm{~J}=7.4 \mathrm{~Hz}, \mathrm{CH}_{2} \mathrm{CH}_{3}\right), 1.85,\left(\mathrm{~m}, 2 \mathrm{H}, \mathrm{CH}_{2} \mathrm{CH}_{3}\right), 4.80,\left(\mathrm{t}, 1 \mathrm{H},{ }^{3} \mathrm{~J}=6.6 \mathrm{~Hz}\right.$, $\mathrm{CHOH}), 6.97\left(2 \mathrm{~d}, 2 \mathrm{H},{ }^{3} \mathrm{~J}=2.9\right.$ and $3.3 \mathrm{~Hz}$, thienyl $\mathrm{H} 3$ and $\left.\mathrm{H} 5\right), 7.21\left(1 \mathrm{H}\right.$, dd, ${ }^{3} \mathrm{~J} 2.9$ and $3.3 \mathrm{~Hz}$, thienyl H4). ${ }^{13} \mathrm{C}$ NMR $\delta 10.1,32.2,71.6,123.7,124.4,126.5$ and 148.7 ppm. MS: $142\left(\mathrm{M}^{\cdot+}\right)$, 124, $113(100 \%), 97,85,57,51,45$. TLC; $\mathrm{R}_{\mathrm{f}}=0.23$ (pentane : $\mathrm{Et}_{2} \mathrm{O}, 3: 1$ ).

1-(2-Thienyl)butanol (3b). Yield: $83.6 \%$. ${ }^{1} \mathrm{H}$ NMR $\delta 0.93\left(\mathrm{t}, 3 \mathrm{H},{ }^{3} \mathrm{~J}=7.4 \mathrm{~Hz}, \mathrm{CH}_{2} \mathrm{CH}_{3}\right), 1,40$ $(\mathrm{m}, 2 \mathrm{H}), 1.80(\mathrm{~m}, 2 \mathrm{H}), 4.87\left(\mathrm{t}, 1 \mathrm{H},{ }^{3} \mathrm{~J}=6.7 \mathrm{~Hz}, \mathrm{CHOH}\right), 6.93(2 \mathrm{~d}, 2 \mathrm{H}$, thienyl $\mathrm{H} 3$ and H5), 7.17 $\left(2 \mathrm{~d},{ }^{3} \mathrm{~J}=3.8\right.$ and $3.9 \mathrm{~Hz}$, thienyl H4) ppm. ${ }^{13} \mathrm{C}$ NMR $\delta 13.8,19.0,41.4,70.0,123.6,124.4,126.5$, 149.0 ppm. MS: $156\left(\mathrm{M}^{\circ+}\right), 138,123,113(100 \%), 97,85,45$. TLC; $\mathrm{R}_{\mathrm{f}}=0.32$ (pentane : Et $2 \mathrm{O}, 3$ : 1).

1-(2-Thienyl)pentanol (4b). Yield: $70.2 \%$. ${ }^{1} \mathrm{H}$ NMR $\delta 0.89\left(\mathrm{t}, 3 \mathrm{H},{ }^{3} \mathrm{~J}=7.0 \mathrm{~Hz}, \mathrm{CH}_{2} \mathrm{CH}_{3}\right), 1.25$ $1.40(\mathrm{~m}, 4 \mathrm{H}), 1.80(\mathrm{~m}, 2 \mathrm{H}), 4.85\left(\mathrm{t}, 1 \mathrm{H},{ }^{3} \mathrm{~J}=6.6 \mathrm{~Hz}, \mathrm{CHOH}\right), 6.92(2 \mathrm{~d}, 2 \mathrm{H}), 7.20\left(2 \mathrm{~d},{ }^{3} \mathrm{~J}{ }^{\prime}=\right.$ $3.4^{\prime}$ and $3.5 \mathrm{~Hz}$ ) ppm. ${ }^{13} \mathrm{C}$ NMR $\delta 14.0,22.5,28.0,38.0,70.2,123.6,124.3,126.5,148.7$ ppm. MS: $170\left(\mathrm{M}^{\circ+}\right), 152,129,113,110,97,85,79,45$. TLC; $\mathrm{R}_{\mathrm{f}}=0.34$ (pentane $\left.: \mathrm{Et}_{2} \mathrm{O}, 4: 1\right)$.

Gram-scale resolutions $(1.5-3 \mathrm{~g})$ were performed in the solvent that gave the best result (high E-value and short reaction times) in small-scale transesterifications.

(R)-1-(2-Thienyl)ethyl butanoate [(R)-1c]. Racemic 1-(2-thienyl)ethanol (1b) (1.5 g) was dissolved in hexane $(50 \mathrm{~mL})$, vinyl butanoate (5 equivalents) was added and the reaction was started by adding immobilised CALB $(200 \mathrm{mg})$ at $30^{\circ} \mathrm{C} / 200 \mathrm{rpm}$. The reaction was monitored by chiral GLC. After $2.5 \mathrm{~h}$ the reaction virtually stopped and the enzyme was filtered off. The enantiomeric excess of both the produced ester and the remaining alcohol was $99 \%$ or better. The ester and the alcohol were separated by column chromatography (EtOAc : hexane; $1: 5$ ), yield $0.91 \mathrm{~g}(78 \%) .{ }^{1} \mathrm{H}$ NMR $\delta 0.95\left(\mathrm{t}, 3 \mathrm{H},{ }^{3} \mathrm{~J}=7.3 \mathrm{~Hz}, \mathrm{CH}_{2} \mathrm{CH}_{3}\right), 1.64(\mathrm{~m}, 5 \mathrm{H}), 2.31\left(\mathrm{t}, 2 \mathrm{H},{ }^{3} \mathrm{~J}=\right.$ $6.9 \mathrm{~Hz}), 6.19\left(\mathrm{q}, 1 \mathrm{H},{ }^{3} \mathrm{~J}=6.6 \mathrm{~Hz}\right), 6.97\left(2 \mathrm{~d}, 1 \mathrm{H},{ }^{3} \mathrm{~J}=3.3 \mathrm{~Hz}\right.$ and $\left.5.1 \mathrm{~Hz}\right), 7.05\left(\mathrm{~d}, 1 \mathrm{H},{ }^{3} \mathrm{~J}=3.3\right.$ $\mathrm{Hz}), 7.27(\mathrm{~d}, 1 \mathrm{H}, 3 \mathrm{~J}=5.1 \mathrm{~Hz}) \mathrm{ppm} .{ }^{13} \mathrm{C} \mathrm{NMR} \delta 14.1,18.9,22.5,36.9,67.8,125.1,125.5,127.0$, 145,2 and 173.2 ppm. MS: $198\left(\mathrm{M}^{\circ+}\right), 128,111,110,85,77,71,45 .[\alpha]_{D}^{20}=+107.2^{\circ}(\mathrm{c} 3.63$, $\mathrm{CHCl3}),+86.8^{\circ}$ (c 4.72, EtOH).

(S)-1-(2-Thienyl)ethanol [(S)-1b]. Physical properties as for (rac)-1b, yield $0.47 \mathrm{~g}(63 \%)$, = $21.3^{\circ}\left(\mathrm{c} 1.88, \mathrm{CHCl}_{3}\right),-14.4^{\circ}$ (c $\left.1.88, \mathrm{EtOH}\right)$.

(R)-1-(2-Thienyl)propyl butanoate [(R)-2c]. Resolution as for 1c. ${ }^{1} \mathrm{H}$ NMR $\delta 0.94(\mathrm{~m}, 6 \mathrm{H})$, $1.66(\mathrm{~m}, 2 \mathrm{H}), 1.95(\mathrm{~m}, 2 \mathrm{H}), 2.31\left(\mathrm{t}, 3 \mathrm{H},{ }^{3} \mathrm{~J}=6.9 \mathrm{~Hz}\right), 6.00\left(\mathrm{t}, 1 \mathrm{H},{ }^{3} \mathrm{~J}=6.9 \mathrm{~Hz}, \mathrm{CHOH}\right), 6.96(2 \mathrm{~d}$, $1 \mathrm{H},{ }^{3} \mathrm{~J}=3.5$ and $\left.3.4 \mathrm{~Hz}\right), 7.04\left(\mathrm{~d}, 1 \mathrm{H},{ }^{3} \mathrm{~J}=3.3 \mathrm{~Hz}\right), 7.25\left(\mathrm{~d}, 1 \mathrm{H},{ }^{3} \mathrm{~J}=3.4 \mathrm{~Hz}\right) \mathrm{ppm} .{ }^{13} \mathrm{C}$ NMR $\delta$ $10.5,14.1,18.9,30.0,36.9,72.7,125.7,126.1,127.0,144.1$ and 173.4 ppm. MS: $212\left(\mathrm{M}^{\circ+}\right), 183$, $142,125,124,123,113,97,71,43 .[\alpha]^{20}=+111.6^{\circ}\left(\mathrm{c} 5.09, \mathrm{CHCl}_{3}\right)$

(S)-1-(2-Thienyl)propanol[ (S)-2b]. Physical properties as for $(r a c)-2 b .[\alpha]^{20}{ }_{D}=-25.4^{\circ}$ (c $\left.1.26, \mathrm{CHCl}_{3}\right)$.

(R)-1-(2-Thienyl)butyl butanoate[(R)-3c). Resolution as for 1c. ${ }^{1} \mathrm{H}$ NMR $\delta 0.95(\mathrm{~m}, 6 \mathrm{H}), 1.38$ $(\mathrm{m}, 2 \mathrm{H}), 1.67(\mathrm{~m}, 2 \mathrm{H}), 1.98(\mathrm{~m}, 2 \mathrm{H}), 2.31\left(\mathrm{t}, 2 \mathrm{H},{ }^{3} \mathrm{~J}^{\prime}=6.9 \mathrm{~Hz}\right), 6.09\left(\mathrm{t}, 1 \mathrm{H},{ }^{3} \mathrm{~J}=6.9 \mathrm{~Hz}\right), 6.97$ 
$\left(2 \mathrm{~d}, 1 \mathrm{H},{ }^{3} \mathrm{~J}=3.5\right.$ and $\left.5.0 \mathrm{~Hz}\right), 7.03\left(\mathrm{~d}, 1 \mathrm{H},{ }^{3} \mathrm{~J}=3.5 \mathrm{~Hz}\right), 7.27\left(\mathrm{~d}, 1 \mathrm{H},{ }^{3} \mathrm{~J}=5.0 \mathrm{~Hz}\right) .{ }^{13} \mathrm{C}$ NMR $\delta$ $14.1,18.6,19.0,19.4,36.2,39.0,71.2,125.0,125.8,126.1,144.4$ and 173.4 ppm. MS: 226 $\left(\mathrm{M}^{\bullet+}\right), 183,156,138,123,110,98,71,43 .[\alpha]^{20}{ }_{\mathrm{D}}=+66.0^{\circ}\left(\mathrm{c} 5.00, \mathrm{CHCl}_{3}\right)$.

\section{Conversion of $(R)-1-(2-T h i e n y l) b u t y l$ butanoate $[(R)-3 c]$ into $(R)$-4-octanol}

$(R)$-1-(2-Thienyl)butyl butanoate $[(R)-3 c]$ was hydrolysed by $\mathrm{K}_{2} \mathrm{CO}_{3}$ in $\mathrm{MeOH}$ to give $(R)-1-(2-$ thienyl)butanol [(R)-3b]. (R)-3b (100 mg) was dissolved in $\mathrm{MeOH}(10 \mathrm{~mL})$ and a suspension of Raney-Ni in water $(2 \mathrm{~mL})$ was added. $\mathrm{H} 2$ was added via a balloon and stirring was continued over night. The solution was filtered and evaporated, the residue was mixed with $\mathrm{NaHCO}_{3}$ solution $(5 \%, 5 \mathrm{~mL})$ and extracted with $\mathrm{CH}_{2} \mathrm{Cl} 2(3 \times 5 \mathrm{~mL})$. The combined extracts were dried and evaporated to give $(R)-4$-octanol $\left(80 \mathrm{mg}, 96.3 \%\right.$ yield). $[\alpha]_{\mathrm{D}}^{20}=+0.25^{\circ}$ (c 1.60, EtOH).

(S)-1-(2-Thienyl)butanol [(S)-3b]. Physical properties as for (rac)-3b. $[\alpha]^{20}{ }_{\mathrm{D}}=-51.5^{\circ}$ (c 1.00 , $\left.\mathrm{CHCl}_{3}\right)$.

(R)-1-(2-Thienyl)pentyl butanoate [(R)-4c]. Resolution as for 1c. ${ }^{1} \mathrm{H}$ NMR $\delta 0.92(\mathrm{~m}, 6 \mathrm{H}), 1.33$ $(\mathrm{m}, 4 \mathrm{H}), 1.65(\mathrm{~m}, 2 \mathrm{H}), 1.93(\mathrm{~m}, 2 \mathrm{H}), 2.28\left(\mathrm{t}, 2 \mathrm{H},{ }^{3} \mathrm{~J}=6.9 \mathrm{~Hz}\right), 6.04\left(\mathrm{t}, 1 \mathrm{H},{ }^{3} \mathrm{~J}=7.0 \mathrm{~Hz}\right), 6.94(2 \mathrm{~d}$, $1 \mathrm{H},{ }^{3} \mathrm{~J}=3.5$ and $\left.5.2 \mathrm{~Hz}\right), 7.03\left(\mathrm{~d}, 1 \mathrm{H},{ }^{3} \mathrm{~J}=3.5 \mathrm{~Hz}\right), 7.25\left(\mathrm{~d}, 1 \mathrm{H},{ }^{3} \mathrm{~J}=5.0 \mathrm{~Hz}\right) .{ }^{13} \mathrm{C} \mathrm{NMR} \delta 13.4$, 13.7, 18.2, 22.1, 27.4, 35.9, 36.2, 70.8, 124.8, 125.4, 126.2, 143.7, 172.7 ppm. MS: $240\left(\mathrm{M}^{\circ+}\right)$, $183,170,152,123,110,97,71,60,43 .[\alpha]_{D}^{20}=+44.4^{\circ}\left(\mathrm{c} 4.01, \mathrm{CHCl}_{3}\right)$.

(S)-1-(2-Thienyl)pentanol [(S)-4b]. Physical properties as for (rac)-4b. $[\alpha]^{20}=-17.9^{\circ}$ (c 1.00 , $\left.\mathrm{CHCl}_{3}\right)$.

Methanolysis of $\boldsymbol{R}-(1 \mathrm{c})$.

$\boldsymbol{R}$-1-(2-Thienyl)ethanol [(R)-1b]. The enantiopure ester(R)-1c (390 mg, $1.97 \mathrm{mmol})$ was added to $15 \mathrm{~mL}$ of hexane. Methanol $(2 \mathrm{~mL})$ and immobilized CALB (30 mg) were added to the reaction mixture and the reaction was left on a shaking incubator at $30^{\circ} \mathrm{C}$ for $72 \mathrm{~h}$. When normal GLC showed complete reaction, the enzyme was removed by filtration and solvent and the formed methylbutanoate was removed by rotavapor. The enantiomeric excess of (R)-1b was controlled by chiral GLC and showed the same ee as the ester ( $>99)$ and the product used without further purificarion.. Yield $238 \mathrm{mg}, 94.3 \%$. $[\alpha]^{20}{ }_{\mathrm{D}}=+21.66^{\circ}$ (c 3.37, $\mathrm{CHCl}_{3}$ ).

\section{Acknowledgments}

We are grateful to The Research Council of Norway (NFR) for financial support. We also thank Novozymes, Bagsværd, Denmark for a gift of CALB.

\section{References}

1. Chen, C.-S.; Fujimoto, Y.; Girdaukas, G.; Sih, C. J. J. Am. Chem. Soc. 1982, 104, 7294.

2. Fantin, G.; Fogagnolo, M.; Medici, A.; Pedrini, P.; Poli, S.; Gardini, F. Tetrahedron: Asymmetry 1993, 4, 1607. 
3. Fantin, G.; Fogagnolo, M.; Medici, A.; Pedrini, P.; Poli, S.; Sinigaglia, M. Tetrahedron: Lett. 1993, 34, 883.

4. Fantin, G.; Fogagnolo, M.; Guerzoni, M. E.; Medici, A.; Pedrini, P.; Poli, S. J. Org. Chem. 1994, 59, 924.

5. Kang, S.-K.; Jeon, J.-H.; Yamaguchi, T.; Kim, J.-S.; Ko, B.-S. Tetrahedron: Asymmetry 1995, 6, 2139.

6. Prasad, K. R. K.; Joshi, N. N. Tetrahedron: Asymmetry 1997, 8, 173.

7. Nishibayashi, Y.; Singh, J. D.; Segawa, K.; Fukazawa, S.-I.; Uemura, S. J. Chem. Soc. Chem. Commun. 1994, 1375.

8. Janssen, P. A. J. US Patent 2,979,507 1961.

9. Jacobsen, E. E.; Hoff, B. H.; Anthonsen, T. Chirality 2000, 12, 654.

10. Noe, C. R.; Knollmüller, M.; Dungler, K.; Miculka, C.; Gärtner, P., Monatshefte für Chemie 1991, 122, 705.

11. Hoff, B. H.; Anthonsen, T. Chirality 1999, 11, 760.

12. Nakamura, K.; Matsuda, T. J. Org. Chem. 1998, 63, 8957.

13. Anthonsen, H. W. E\&K Calculator version 2.03. http://Bendik.chembio.ntnu.no 1996.

14. Liu, H.-L.; Hoff, B. H.; Anthonsen, T. Chirality 2000, 12, 26. 